INPLASY

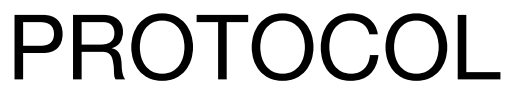

To cite: Huang et al. Manual therapy for idiopathic scoliosis: a protocol for systematic review and meta-analysis. Inplasy protocol 202070058. doi:

10.37766/inplasy2020.7.0058

Received: 15 July 2020

Published: 15 July 2020

Corresponding author: Lingjun Kong

chunyong01@163.com

Author Affiliation:

Yueyang Hospital of Integrated

Traditional Chinese and Western Medicine, Shanghai University of Traditional Chinese Medicine

Support: Lianyungang hospital of TCM

Review Stage at time of this submission: Preliminary searches.

Conflicts of interest:

There is no conflicts of interest.

\section{Manual therapy for idiopathic scoliosis: a protocol for systematic review and meta-analysis}

Huang, Q13 Zhang, L2; Kong, L³.

Review question / Objective: The effects of manual therapy in the management of idiopathic scoliosis on patient-centered outcomes (such as aesthetics, pain, disability, quality of life, etc.), and secondarily on radiographic outcomes (Cobb angle, vertebral rotation, etc.).

Condition being studied: More patients with idiopathic scoliosis (IS) preferred to choose manual therapy as a complementary conservative treatment, but the effects of manual therapy for IS remains controversial. The previous reviews could not draw reliable conclusion due to few eligible studies to perform a meta-analysis. In the last decade, however, several new studies were published that assessed the effects of manual therapy in the management of IS, especially in China. Therefore, the present systematic review and meta-analysis will be performed to examine whether manual therapy is effective for IS primarily in improving patient-centered symptoms and secondarily in radiographic outcomes.

INPLASY registration number: This protocol was registered with the International Platform of Registered Systematic Review and Meta-Analysis Protocols (INPLASY) on 15 July 2020 and was last updated on 15 July 2020 (registration number INPLASY202070058).

\section{INTRODUCTION}

Review question / Objective: The effects of manual therapy in the management of idiopathic scoliosis on patient-centered outcomes (such as aesthetics, pain, disability, quality of life, etc.), and secondarily on radiographic outcomes (Cobb angle, vertebral rotation, etc.). 
Condition being studied: More patients with idiopathic scoliosis (IS) preferred to choose manual therapy as a complementary conservative treatment, but the effects of manual therapy for IS remains controversial. The previous reviews could not draw reliable conclusion due to few eligible studies to perform a meta-analysis. In the last decade, however, several new studies were published that assessed the effects of manual therapy in the management of IS, especially in China. Therefore, the present systematic review and meta-analysis will be performed to examine whether manual therapy is effective for IS primarily in improving patient-centered symptoms and secondarily in radiographic outcomes.

\section{METHODS}

Search strategy: A computerized literature search will be performed in the following electronic databases from their inceptions to June 2020: PubMed, EMBASE, MEDLINE, Cochrane Central Register of Controlled Clinical Trials, China Knowledge Resource Integrated Database, Wanfang Data Information, and Weipu Database for Chinese Technical Periodicals. The following key terms will be used in combination to develop search strategy for each electronic database: (scoliosis OR spinal curve) AND (manual therapy OR massage OR chiropractic OR osteopathy OR mobilization OR spinal manipulation OR myofascial release OR Tuina OR Shiatsu). The literature search strategy is summarised for PubMed in Table 1. Manual searching will be conducted at the library of Shanghai university of traditional Chinese medicine. The reviewers will screen the reference lists of eligible studies and relevant reviews to identify additional sources of information.

Participant or population: Participants with a diagnosis of idiopathic scoliosis, spinal disorder of unknown cause at least a $10^{\circ}$ lateral curve of the spine.

Intervention: The eligible intervention is manual therapy, is a skilled hand manipulation, including massage, chiropractic, osteopathy, mobilization, spinal manipulation, myofascial release, Tuina, Shiatsu, etc.

Comparator: The control interventions will include observation, bracing, exercise therapy, medicine, education, and any treatments without manual therapy. The included studies may assess the effects of manual therapy compared with other therapy (such as manual therapy versus bracing). The study valuating the effects of manual therapy plus a conservative intervention compared with the same conservative intervention (such as manual therapy plus bracing versus bracing) will also be included.

Study designs to be included: Randomized controlled trials.

Eligibility criteria: The randomized controlled trial assessed the effects manual therapy for idiopathic scoliosis primarily on patient-centered outcomes (such as aesthetics, pain, disability, quality of life, etc.) and secondarily on radiographic outcomes (Cobb angle, vertebral rotation, etc.).

Information sources: Electronic databases from their inceptions to June 2020 to identify randomized controlled trials of manual therapy in the management of IS: PubMed, EMBASE, MEDLINE, Cochrane Central Register of Controlled Clinical Trials, China Knowledge Resource Integrated Database, Wanfang Data Information, and Weipu Database for Chinese Technical Periodicals.

Main outcome(s): Primarily outcomes on patient-centered outcomes (such as aesthetics, pain, disability, quality of life, etc. ).

Additional outcome(s): Secondarily outcomes on radiographic outcomes (Cobb angle, vertebral rotation, etc.).

Quality assessment / Risk of bias analysis: The quality of the included studies will be assessed independently by two reviewers using the Physiotherapy Evidence 
Database (PEDro) scale. The overall quality of evidence was assessed using the Grades of Recommendation, Assessment, Development and Evaluation (GRADE) framework including the risk of bias, inconsistency, indirectness, imprecision, and publications bias. Any disagreement will be resolved through discussion among reviewers.

Strategy of data synthesis: The metaanalysis will be performed with the Review Manager Version 5.3 software. For the continuous data, the changes from baseline will be used in the meta-analysis. $A$ random effects model will be used for a better analysis of the clinical heterogeneity. If outcome measure scales are the same, the mean difference (MD) and $95 \%$ confidence intervals $(\mathrm{Cl})$ will be calculated. In the case of different outcome measure scales, the standardized mean difference (SMD) and $95 \% \mathrm{Cl}$ will be calculated. According to the recommendations of the Cochrane handbook for systematic reviews of interventions, the heterogeneity will be assessed using 12 statistic and Cochran $Q$ statistic.

Subgroup analysis: The subgroup analysis will be conducted based on different control interventions and subpopulations if there are more than three eligible studies.

Sensibility analysis: Sensibility analysis will be performed by evaluating the changes of pooled effect size after each study was eliminated one by one.

Country(ies) involved: China.

Keywords: manual therapy, idiopathic scoliosis, meta-analysis, systematic review.

Contributions of each author:

Author 1 - Qian Huang - Conceptualization, funding acquisition, investigation, methodology, project administration, supervision, writing - original draft.

Author 2 - Lei Zhang - Investigation, methodology, writing -original draft

Author 3 - Lingjun Kong Conceptualization, funding acquisition, investigation, supervision, writing -review and editing. 